\title{
EXTRACELLULAR MATRICES IN GYNECOLOGICAL SURGERY - LITERATURE REVIEW
}

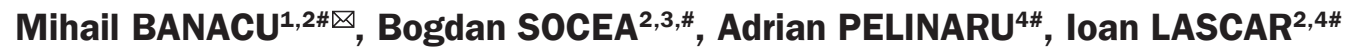 \\ 1 „St. Pantelimon“ Emergency Clinical Hospital, Obstetrics and Gynecology Department, Bucharest, \\ Romania \\ 2 "Carol Davila“ University of Medicine and Pharmacy, Bucharest, Romania \\ 3 „St. Pantelimon“ Emergency Clinical Hospital, Surgical Department, Bucharest, Romania \\ ${ }^{4}$ Clinical Emergency Hospital of Bucharest, Clinic of Plastic Surgery, Aesthetic and Reconstructive \\ Microsurgery, Bucharest, Romania
}

\#These authors contributed equally

Received 03 May 2020, Accepted 18 May 2020

https://doi.org/10.31688/ABMU.2020.55.2.14

\section{Abstract}

The use of alloplastic materials in different surgical urogynecological interventions has been subject to limitations during the past years by the international societies, because of late onset mesh-related complications. Xenograft-based biomaterials obtained from extracellular matrix offer a proper acellular foundation for tissue repair in urogynecology, with improved tissue-graft rejection. We have searched PubMed database to identify all publications regarding the use of extracellular matrix in gynecological reconstructive surgery. The use of these matrices in organ prolapse surgery has shown efficient integration and favorable outcomes. Biomaterials are used for vaginoplasties in patients with vaginal congenital agenesis, patients who underwent vaginal radical resection for cervical cancer or patients diagnosed with profound endometriosis, who had partial vaginal resection. Biomaterials may also have a future in vaginoplasties performed for aesthetic reasons. It is foreseen that in the future these

\section{Résumé}

Matrices extracellulaires en chirurgie gynécologique - revue de la littérature

L'utilisation de matériaux alloplastiques dans différentes interventions chirurgicales en génito-urologie a été soumise à des limitations au cours des dernières années par les sociétés internationales, en raison de complications tardives liées au maillage. Les biomatériaux à base de xénogreffe obtenus à partir de la matrice extracellulaire offrent une base acellulaire appropriée pour la réparation tissulaire en génito-urologie, avec un rejet amélioré de la greffe tissulaire. Nous avons étudié la base de données PubMed afin d'identifier toutes les publications concernant l'utilisation de la matrice extracellulaire en chirurgie reconstructive gynécologique. L'utilisation de ces matrices en chirurgie du prolapsus d'organe a montré une intégration efficace et des résultats favorables. Les biomatériaux sont utilisés pour les vaginoplasties réalisées chez des $\bowtie$ Address for correspondence:
Mihail BANACU

„St. Pantelimon“ Emergency Clinical Hospital, Obstetrics and Gynecology Department, Bucharest, Romania

Address: Pantelimon street no 340-342, Bucharest, Romania

Email: mbanacu@gmail.com; Phone: +40722306013 
biomaterials might be used in the treatment of certain fetal structural anomalies, like neural tube defects. Although nowadays the use of biological grafts is quite limited, due to the lack of prospective randomized trials to compare them with classical surgical techniques, with the emerging technologies, in the near future we foresee its significant role in urogynecology, plastic surgery repair and possibly fetal congenital malformation correction.

Keywords: extracellular matrices, urogynecology, vaginoplasty, biological graft.

\section{List of abbreviations:}

ADM - acellular dermal matrix

ECM - extracellular matrix

MRKH - Mayer-Rokitansky-Küster-Hauser Syndrome

MNC - mononuclear cells

MSC - mesenchymal stem cells

AF-MSC - amniotic fluid-derived mesenchymal stem cells

PD - porcine dermis

PRGF - platelet rich growth factors

$\mathrm{RF}$ - rectus abdominis autologous fascia

SIS - Porcine small intestinal submucosa

SSM - skin sparing mastectomy

TOT - Trans obturator tape

UBM - urinary bladder matrix

VAS - visual analogue scale

During the last few years, urogynecological surgery suffered a set-back because of limitations introduced by international societies regarding the use of alloplastic materials. Although the use of polypropylene mesh for the correction of urinary incontinence and organ prolapse had satisfactory results on short-term and standardization of the procedure later reduced intra- and post-op complications, a significant number of patients developed long-term complications. Of these erosions, chronic pain and dyspareunia generated severe interference with the patients' quality of life, raised concerns with respect to safety and thus limited the use of mesh in all gynecological surgical fields, until further safety studies.

Biological materials based on xenografts are either extracellular matrices (ECM) obtained from porcine small intestinal submucosa (SIS) or porcine acellular dermal matrix (ADM). They form efficient acellular resistance structures, which are also flexible and proper for tissue repair.

This technology is based on the extracellular matrix, a structure found in most body tissues, support for intercellular signaling and tissue integration through renewal. In time, this structure is fully patientes diagnostiquées avec une agénésie congénitale vaginale, des patientes ayant subi une résection radicale vaginale pour un cancer du col utérin ou des patientes diagnostiquées avec une endométriose profonde qui ont subi une résection vaginale partielle. Les biomatériaux peuvent également avoir un avenir dans les vaginoplasties réalisées pour des raisons esthétiques. Il est prévu qu'à l'avenir ces biomatériaux pourraient être utilisés dans le traitement de certaines anomalies structurales foetales comme les anomalies du tube neural. Bien que de nos jours, l'utilisation de greffes biologiques soit assez limitée en raison du manque d'essais prospectifs randomisés pour les comparer aux techniques chirurgicales classiques, avec les technologies émergentes, dans un avenir proche, nous prévoyons son rôle important dans la génito-urologie, la chirurgie plastique réparatrice et éventuellement la correction de malformations congénitales fœetale.

Mots-clés: matrice extracellulaire, génito-urologie, vaginoplastie, greffe biologique.

accepted within the tissue, offering resistance and at the same time remodeling, with similar properties to a healthy functional tissue. Its use is simple and highly effective. Extracellular matrix is composed of proteins responsible for the actual structure (collagen and elastin), glycoproteins (fibronectin and laminin), glycosaminoglycans and proteoglycans (heparan sulfate, heparin, hyaluronic acid), other proteins like thrombospondin, osteospondin and tenascin. The most abundant molecule is collagen, which offers structure to the matrix, while glycosaminoglycans and proteoglycans absorb water and attract growth factors, later used for cellular signaling.

These extracellular matrices are obtained by decellularization, a process leading to resistant new matrices which can support future cell culture proliferation. The process of decellularization, as well as that of disinfection and inactivation, are compulsory to assure safe usage and to diminish graft rejection.

We performed a literature review on surgical alternative options using new materials (xenografts) with lower rejection potential and higher capacity for integration, with the purpose of being used in all 
urogynecological surgical fields, as well as in aesthetic and plastic surgical gynecology.

The role of extracellular matrices in tissue recovery consists in offering a structure that can attract and support cell populations using different signaling pathways. In addition to having a specific architecture, they improve tissue repair capacities. Thus, we intend to investigate whether the use of these materials in plastic surgery, for correction of vaginal pathologies or as a structure of resistance in genital prolapse surgery, represents an option.

We searched the PubMed database for all articles published in the last 20 years (2000-2019), referring to human or animal studies associated with the gynecological field, using the following keywords: biomatrix, extracellular matrix, porcine intestinal submucosa, acellular dermal matrix. The search resulted in 46 articles; from these, 20 articles referred to studies conducted on animals and 26 articles on human studies.

The use of synthetic polypropylene mesh for the treatment of uterine prolapse has already been well established, due to its long-lasting resistance properties and reduced risk of relapse. However, during the last decade, concerns about safety were raised, and in the last years there is a widespread conservative attitude towards its usage in many European countries, the United States of America and the Australia. Biological acellular grafts, like extracellular matrices, represent an option in this situation.

Culligan et al published a prospective randomized study, comparing sacral colpopexy using porcine dermis (PD) with synthetic polypropylene mesh, in cases of uterine prolapse ${ }^{1}$. A total of 115 patients were enrolled in the study and at 12-month postoperative follow-up there were no significant differences between the two groups regarding the healing rate (defined as stage 1 prolapse), of $84.2 \%$ for PD graft and $89.7 \%$ for the polypropylene mesh. No important operative complications were registered. Another study published in 2016 by $\mathrm{Cao}^{2}$ evaluated the use of these materials in vaginal prolapse surgery. In this study, 40 patients diagnosed with vaginal prolapse, because of anterior and posterior vaginal wall defects, were enrolled. All of them had surgery using ECM of SIS. At 12-month postoperative follow-up, the subjective recurrence rate compared to the objective recurrence rate was $7.5 \%$ to $40 \%$. No postoperative erosions were identified. In the same study, it was noted that the recurrence of the anterior compartment prolapse needs an alternative surgical option and long-term follow-up is compulsory to be able to draw definitive conclusions.

Anterior and posterior vaginal wall prolapse, especially those diagnosed after vaginal deliveries, are common perineal dysfunctions. There are few surgical options available to treat these dysfunctions, because of urinary bladder, urethra and rectum proximity, as well as other pelvic organs that might interfere with each technique. Consequently, the use of synthetic materials is reduced because of increased rejection risk, chronic pain, erosions, perforations and fistulas.

A new surgical technique using ECM from SIS (Biodesign $^{\mathrm{TM}}$; Cook Medical Inc., Bloomington, IN, USA) substitution material for the correction of prolapse is described in Liang's study published in $2016^{3}$. The correction of anterior and posterior vaginal compartment was performed in all 18 patients enrolled in this study. Follow-up was made up to 33 months postoperatively. Less scarring tissue, smooth vaginal walls and increased resistance were reported.

In 2014, a prospective randomized trial, published by Robert ${ }^{4}$, brought into attention the use of SIS in the surgery of anterior vaginal compartment. This study compared the functional results, evaluated 12 months after surgery, for anterior vaginal wall prolapse in patients who benefited from SIS mesh with patients who received native tissue. There were 28 patients who received mesh and 29 who received tissue. About $56 \%$ of patients in the mesh group were healed by the 1-year follow-up compared to $61 \%$ in the tissue group. Regarding recurrence rate and persistence of the prolapse, no significant differences were seen between the two groups. Chronic pain was quantified in 4 patients from the mesh group and in 3 patients from the tissue group. The study concluded that at 12-month follow-up there were no statistical differences between mesh and native tissue surgical repairs.

Before that, in 2006, a case-control study conducted by Chaliha ${ }^{5}$ on 28 patients focused on the effect of surgical treatment of anterior vaginal wall prolapse on patients' quality of life. The study aimed to compare classic colporrhaphy to surgery using SIS graft. In each group, 14 patients were enrolled and underwent surgery with the assigned technique. After 6 and 24 months, all enrolled patients came for evaluation and received 2 validated questionnaires: one POP-Q and one on life quality assessment. Results showed that at 6 months after surgery, patients in SIS group had a statistically significant improvement of life quality, based on all measured indicators. The same group marked significant improvement on all objective measurements, made via POP-Q, except for total vaginal length. At 2-year follow-up, there were no statistically significant differences between life quality aspects measured in both groups ${ }^{5}$.

With respect to sexual function after vaginal repair surgery, Feldner et al stated in $2012^{6}$ that there 
were no differences regarding libido, orgasm achievement, sexual satisfaction and dyspareunia 12 months after surgery between study groups. The comparison was made between classic colporrhaphy and SIS graft surgery, just as in Chaliha's 2006 study. Both surgical procedures improved sexual life quality, with no significant differences being noted.

Synthetic polypropylene meshes are also of use in the surgical treatment of urinary incontinence when trans obturator tape (TOT) technique is applied. The efficiency of ECM from SIS for TOT procedure was observed by Wang et $\mathrm{al}^{7}$ in 42 operated patients. The main structure of this study was based on follow-up at 1 year and 5 years, respectively. Surgical results on urodynamic function and a questionnaire for objective measurement of surgical success were assessed. Thirty-four of all 42 patients had significant relief of urinary incontinence symptoms at 1-year follow-up visit. Four cases of graft rejection were registered, but with no major complications. However, no notable changes were obtained in urodynamic parameters. Furthermore, in the long-term, SIS grafts curative potential proved to be lower compared to that of synthetic materials.

Complications related to graft rejection in vaginal and prolapse surgery are meant to be reduced by using SIS substitution implants. A case report on a patient with graft rejection after TOT procedure performed for urinary incontinence was published in 2009 by Wang ${ }^{8}$. The complication was initially approached by debridement and material excision, but no favorable outcome was obtained. After prednisone administration, the expected outcome was finally reached.

In urogynecology, complications following polypropylene mesh use are already well-known. Erosion of the mesh as a foreign body through the vagina is the most debilitating one, resulting in high morbidity. When there is an important loss defect and the surrounding tissue cannot compensate, biological grafts are useful options to consider. In literature, Khong published in 2007 nine case reports, in whom polypropylene mesh eroded in the vagina'. ECM from SIS (Surgisis ${ }^{\circledR}$ ) was used to treat the defect. The erosion complicated the evolution at 12 weeks postoperatively and the mean diameter of the defect was between 1 and $4 \mathrm{~cm}$. After excising the material and replacing it with the graft, 5 patients fully recovered, 3 had persistent erosion and one patient needed another surgical intervention. The authors concluded that ECM from SIS (Surgisis ${ }^{\circledR}$, Cook Medical Inc., Bloomington, IN, USA) can be a therapeutic option in case of large vaginal defects following polypropylene mesh erosion.

Synthetic meshes can also be used for urinary sphincter incontinence. Taskinen compared mesh versus ECM from PD and followed up cases at 1, 6 and 12 months after surgery ${ }^{10}$. Although the surgical technique is easy, it involves high risks when working on normal pelvic anatomy and the results in neuropathic incontinence are promising, the 12-month postoperative data collected were not satisfying at all.

Giri et al studied the use of biological materials in the treatment of urinary incontinence, when applying pubovaginal technique ${ }^{11}$. The study aimed to compare the use of ECM from PD versus rectus abdominis autologous fascia (RF). A number of 101 patients were enrolled: 51 received PD graft and 50 received RF. A specific follow-up protocol was established for 3, 6, 12 and 36 months after surgery. Proceeding with it, researchers summed up that $80.4 \%$ of the patients treated with RF graft reported an enhancement of clinical status or full recovery from the incontinence, while only 54\% from the PD graft met the same results. As a conclusion, the article states that PD grafts are not appropriate to use with pubovaginal procedure.

Toll defined vaginoplasty in 1946 as "the art of plastic correction of urethral, urinary bladder or rectal herniation into the vaginal canal"12. This notion is still used to describe this kind of vaginal reconstruction, but nowadays it also includes other pathologies, like congenital anomalies, vaginal reconstruction after radical surgery for cancers or sex reassignment surgery.

One type of biological material used for this kind of surgery is acellular dermal matrix (ADM). In the medical literature, we found a presentation of 16 cases of cervical cancer treated with radical surgery, who afterwards underwent vaginal reconstruction using $\mathrm{ADM}^{13}$. Objective analysis of patient's sexual satisfaction after the plastic intervention indicated favorable results. Restoring sexual activity 2 months after surgery prevented long-term use of vaginal tutor; all patients had normal vaginal caliber and $75 \%$ declared themselves satisfied with their sexual life quality.

Vaginoplasty for substitution of excised vagina in case of cervical, vaginal or vulvar cancer has long been approached in different studies. Lin et al published a case report where vaginoplasty was performed after vaginectomy in a patient diagnosed with recurrence of cervical cancer at the resection site, 8 years after radical surgery ${ }^{14}$. The correction surgery used ECM from SIS. Afterwards, the patient went to 3 rounds of chemotherapy. Normal sexual activity was restored 8 months after surgery. Her current condition is free of recurrence signs.

Another interesting case report about vaginoplasty with ECM from SIS was presented by Bratila et al, about a patient diagnosed with verrucous carcinoma who had multiple excisional interventions for 
over 11 years and the remaining vagina was successfully corrected with biological grafts ${ }^{15}$. Two months after vaginoplasty, the graft was completely cohesive with the surrounding tissue and full recovery was reported.

A case series of 16 patients with radical surgery combined with radiation therapy for cervical cancer described the implementation of vaginoplasty with ADM ${ }^{13}$. Proper vaginal dimensions were assured by means of a dilator, which also prevented stenotic complications. A subjective and objective assessment at 6 and 12 month post-op revealed that epithelization was observed at only 2 weeks post-op and after 1 year the vaginal length raised from $1.31 \pm 0.4 \mathrm{~cm}$ to $4.13 \pm 0.43 \mathrm{~cm}$. Also, 12 out of 16 patients had satisfactory sexual life one year after surgery.

Digestive cancers like rectal adenocarcinoma with vaginal invasion like the one presented by Bhavsar in a 51-year old patient treated with radical surgery might also imply large vaginal excisions requiring $\mathrm{ADM}^{16}$. Three weeks after surgery, the patient was assessed and granulation tissue was seen covering the ADM graft, while vaginal lumen was still preserved. Furthermore, at 10 months follow-up, no stenosis has been identified and complete vaginal healing was confirmed.

Substitutive surgery for benign pathologies reaches also for vaginoplasty benefits. Endometriosis treatment might require excision of vaginal nodules, leaving an important defect of the vagina, that could need further repair. Lemos et al in 2009 described the role of vaginoplasty in the surgical management of a patient with rectovaginal endometriosis ${ }^{17}$.To minimize the risk of rectovaginal fistula, the nodule was resected in 2 step surgery. The excised vaginal wall was replaced with a SIS graft, useful in the prevention of stenosis or vaginal shortening. The area healed properly and no scar tissue could be detected.

From vaginal mucous tissue to dermal defects, there were more complex studies in the literature, focusing on graft utility in these situations. Park developed in 2014 a retrospective study, comparing cutaneous defect healing after autologous skin graft implementation with or without acellular graft CGDerm and AlloDerm in 78 patients $^{18}$. Nine months after implant, results showed that the study groups had similar healing rates with respect to elasticity, hydration and barrier skin function. However, better aesthetic results were met in the case of ADM grafts. Based on this information, we can estimate that this type of grafts might prove useful in the surgery of vulvar cutaneous defects, either under aesthetic or functional considerations.

An experimental study conducted by Eggink et al in 2006 suggested the possibility to correct cutaneous defects associated with neural tube fetal malformations with biological grafts (SIS and collagen biological grafts $)^{19}$. The experiment was carried out on pregnant ewes, beginning on $79^{\text {th }}$ gestational day. Initially, a cutaneous defect was created in utero on 19 ewes and in 4 controls. In 7 of the 19 ewes, the defect was let open and in the remaining 12 ewes different biological grafts were used to correct the defect: collagen-based graft in 4 animals, skin graft in 3 animals and SIS in 5 animals. After one week of life, all animals were sacrificed and histopathological examination was performed. Results were as follows: spinal bone marrow lesions were detected in all 7 animals with open defect; there were no significant histological differences between the 3-type graft groups. The study concluded that all three types of biological grafts are useful for the correction of ewes' fetal neural tube defect, to prevent spinal bone marrow lesions. Two years later, the same team of researchers conducted by Eggink ran another investigation on ewes, to find out if in utero correction of artificially created spina bifida using collagen biological matrices is possible ${ }^{20}$. This time, 20 ewes were operated between the $72^{\text {nd }}$ and $79^{\text {th }}$ day of gestation; in 15 ewes, a spina bifida defect was created -8 of the ewes had the defect covered in 2 weeks and 7 were left uncorrected; 5 ewes were used as control group. Five cases of corrected spina bifida defect survived and one week after delivery, neurological examination showed that spinal cord function had no alterations and normal architecture was preserved. In the control group, only 4 ewes survived, one having altered spinal cord function, the others showing histological alterations.

The subject concerning restitution of physiological anatomy in patients with congenital malformations like vaginal and or cervical agenesis or hypoplasia, Mayer-Rokitansky-Küster-Hauser Syndrome (MRKH), focuses on adapting surgical techniques to the use of substitution materials made of ECM from SIS. A review from 2014, about surgical and non-surgical correction techniques of vaginal hypoplasia, concluded that in specialty literature there are no prospective, longitudinal and comparative studies which can provide evidence-based information for corresponding guidelines ${ }^{21}$. Non-surgical techniques involving dilation are still preferred, due to their reduced rate of complications, along with high successful registered rate (75\%). Future studies on ECM from SIS will have to focus not only on adequate vaginal length, but also on the complexity of psychological and sexual interconnection encountered in patients, as well as on acceptable approaches regarding patient's psychology in these specific situations.

Robotic reconstruction was also described in literature by Zhang et al, after using SIS graft in 4 
patients with congenital vaginal and cervical atre$\mathrm{sia}^{22}$. An intravaginal tutor was kept 12 months after surgery. Case selection was carefully performed before surgical intervention. All cases had favorable outcomes and normal vaginal caliber. Furthermore, Shen et al compared the vaginal reconstruction using SIS graft with autologous skin graft $^{23}$. There were no differences between the two groups regarding menstrual cycle recommencement; skin graft achieved higher vaginal length in patients who received them. On the other hand, patients from SIS graft group had higher satisfaction over cosmetic results 6 months after surgery. These studies are limited by short post-op follow-up period and the lack of data about sexual life and fertility.

Functional results after vaginoplasty in patients with MRKH were presented in an article by Ding et al, where two different surgical techniques were studied: correction using ECM from SIS graft and laparoscopic peritoneal vaginoplasty $y^{24}$. Operative success was defined as vaginal length $>6 \mathrm{~cm}$ after intervention; functional evaluation was performed using a questionnaire and partner's satisfaction was quantified using visual analogue scale (VAS). Researchers deduced that vaginoplasty using SIS took a shorter time to perform compared to laparoscopic approach. High expenses of SIS techniques were due to ECM. Also, $46 \%$ from SIS group and 29\% from laparoscopic group noted vaginal shortage $<7 \mathrm{~cm}$. Among patients who recovered their sexual activity, satisfaction between partners was approximately the same $\mathrm{e}^{25}$.

An alternative for SIS grafts was proposed by Zhang: porcine vaginal mucosa derived graft destined to improve vaginal reconstruction results ${ }^{26}$. The new ECM was compared with the old one from SIS and promising results associating good biocompatibility were already evidenced.

Improvement of vaginal reconstruction results using ECM from SIS was also demonstrated by adding mesenchymal stem cells (MSC) in a study developed on rodents by Zhang in 2018 ${ }^{27}$. Augmentation of ECM was obtained. MSC managed to accelerate structural and functional integration of the graft. Immune response modulation was proved by another study on rodents, where amniotic fluid-derived mesenchymal stem cells (AF-MSC) were used, concluding that ECM from SIS is an approachable substrate for AF-MSC cultures and that matrix augmentation with AF-MSC does not significantly interfere with the immune response after implantation ${ }^{28}$.

The macrophage role in the process of healing is already well-known, especially considering ECM graft. For assuring a good integration of ECM, a limited immune response is preferred during healing process. Macrophage activity from spinal bone marrow was described in an article, using rodents and human (THP-1 transformed human mononuclear cell line) samples applied over 2 different ECM, one derived from urinary bladder (urinary bladder matrix - UBM) and one from SIS ${ }^{25}$. Results showed different immune responses for the 2 matrices and suggest that macrophage source is essential and important in generating host-specific immune response. This way, the difficulty in predicting therapeutic results is explained.

ADM utility in mammary reconstruction was detailed in a review published by Eichler ${ }^{29}$. From over 7 000 cited reconstructive mastectomies, this graft was most often used in case of skin-sparing mastectomies (SSM). Also, ADM can be used in breast augmentation by applying it in the subpectoral fixation of autologous tissue. High costs limit their applicability, although their potential is very attractive. Mammary reconstructive surgery after breast cancer SSM using ADM graft was also reported by Knabben, in a retrospective study based on 10 selected cases $^{30}$. All cases were evaluated 6 months after surgery. Patient's satisfaction over cosmetic results was exceeded and objective results marked symmetrical coverage of the implant, with favorable aesthetic results.

Biological grafts are also used in tissue engineering. Karolinska Institute conducted a complex research over placental amnion capacity and mononuclear cells (MNC) with thrombocyte-rich growth factors (platelet rich growth factors - PRGF), to form stable biological materials with the support of ECM for further autologous transplant in selected cases of congenital malformations. Preliminary conclusions confirmed that the 2 types of cell cultures are promising sources of autologous tissue ${ }^{31}$.

Other study directions emphasized the importance of ECM source; this way, ECM from young animals is important for remodeling function preservation; ECM from old animals showed alterations in macrophage phenotype ${ }^{32}$.

Vaginal repair surgery, situated at the border of plastic, urologic and gynecological surgery, still represents a complex challenge in achieving satisfying aesthetic and functional results for patients and their partners. Medical practice confronts with delicate, but serious matters, like patients' quality of life and the couple associated with fertility issues.

Randomized trials and long-term observational studies are still lacking; surgical and non-surgical evidence-based management is limited to series of cases and retrospective studies.

It is important to take into consideration the patient's life quality and psychological status, before and after surgical treatment, in order to achieve their full integration in a proper and complete treatment 
scheme, regardless of their pathology, organ dysfunction, congenital malformations, iatrogenic physical and functional alterations following birth process or previous surgical interventions for benign or malignant affections.

The future of biologic materials is bright, in the search for a potent graft for urogynecological use, combining good biocompatibility with remodeling properties and resistance. The study of graft validity should be validated in the future by prospective studies.

\section{Author Contributions:}

M.B. conceived the original draft preparation. M.B. and I.L. were responsible for conception and design of the review. M.B. and A.P. were responsible for the data acquisition. B.S. and M.B. were responsible for the collection and assembly of the articles/published data, and their inclusion and interpretation in this review. M.B., B.S., A.P. and I.L. contributed equally to the present work. All authors contributed to the critical revision of the manuscript for valuable intellectual content. All authors have read and agreed with the final version of the manuscript.

\section{Compliance with Ethics Requirements:}

„The authors declare no conflict of interest regarding this article"

„No funding for this study"

\section{Acknowledgements:}

None

\section{References}

1. Culligan PJ, Salamon C, Priestley JL, Shariati A. Porcine dermis compared with polypropylene mesh for laparoscopic sacrocolpopexy: A randomized controlled trial. Obstet Gynecol. 2013;121:143-151

2. Cao TT, Sun XL, Wang SY, Yang X, Wang JL. Porcine small intestinal submucosa mesh for treatment of pelvic organ prolapsed. Chin Med J (Engl). 2016;129:2603-2609

3. Liang X. A novel technology for vaginal reconstruction using porcine small intestinal submucosa. Plastic and Reconstructive Surgery - Global Open. 2016;4:753

4. Robert M, Girard I, Brennand E, et al. Absorbable mesh augmentation compared with no mesh for anterior prolapse a randomized controlled trial. Obstet Gynecol. 2014;123:288-294

5. Chaliha C, Khalid U, Campagna L, Digesu GA, Ajay B, Khullar V. SIS graft for anterior vaginal wall prolapse repair - A case-controlled study. Int Urogynecol J. 2006;17:492-497

6. Feldner PC, Delroy CA, Martins SB, Castro RA, Sartori MGF, Girão MJBC. Sexual function after anterior vaginal wall prolapse surgery. Clinics. 2012;67:871-875

7. Wang CL, Shen CJ, Lin KL, Long CY. Clinical effects of transobturator tape procedure with porcine small intestine submucosa for female stress urinary incontinence. Kaohsiung J Med Sci. 2016; 32:142-146

8. Wang CL, Hsu CS, Long CY. Graft-versus-host disease following transobturator tape procedure with small intestinal submucosa (Surgisis): A case report. Int Urogynecol J. 2009; 20:1149-1151

9. Khong SY, Lam A. Use of Surgisis mesh in the management of polypropylene mesh erosion into the vagina. Int Urogynecol J. 2011; 22:41-46

10. Taskinen S, Fagerholm R, Rintala R. Mini-invasive collagen sling in the treatment of urinary incontinence due to sphincteric incompetence. Int Braz J Urol. 2007; 33:395-400

11. Giri SK, Hickey JP, Sil D, et al. The long-term results of pubovaginal sling surgery using acellular cross-linked porcine dermis in the treatment of urodynamic stress incontinence. J Urol. 2006; 175:1788-1792

12. Toll RM. Vaginoplasty. Am J Surg. 1946;72(5):742

13. Wang Z, Huang J, Zeng A, Wu M, Wang X. Vaginoplasty with acellular dermal matrix after radical resection for carcinoma of the uterine cervix. J Investig Surg. 2019; 32(2):180-185

14. Lin Y, Zhou J, Dai L, Cheng Y, Wang J. Vaginectomy and vaginoplasty for isolated vaginal recurrence 8 years after cervical cancer radical hysterectomy: A case report and literature review. J Obstet Gynaecol Res. 2017;43(9):1493-1497

15. Bratila E, Bratila CP, Comandasu DE, et al. Perineal reconstruction with biologic graft vulvoplasty for verrucous carcinoma treated by repeated vulvar excisions: A case report. Rom J Morphol Embryol. 2015;56:537-543

16. Bhavsar AK, Lin-Hurtubise KM, Dietrich CS. Restoration of vaginal anatomy after extensive posterior wall resection utilizing human acellular dermal matrix. Gynecol Oncol Reports. 2016;16: 14-16

17. Moreira Lemos NL de B, Kamergorodsky G, Antunes Faria AL, Ayroza Galvão Ribeiro PA, Flores Auge AP, Aoki T. Small intestinal submucosa patch for extensive vaginal endometriosis resection. J Minim Invasive Gynecol. 2009; $16: 765-767$

18. Park JY, Lee TG, Kim JY, Lee MC, Chung YK, Lee WJ. Acellular dermal matrix to treat full thickness skin defects: follow-up subjective and objective skin quality assessments. Arch Craniofacial Surg. 2014;15:14-21

19. Eggink AJ, Roelofs LAJ, Lammens MMY, et al. Histological evaluation of acute covering of an experimental neural tube defect with biomatrices in fetal sheep. Fetal Diagn Ther. 2006; 21(2):210-6

20. Eggink AJ, Roelofs LAJ, Feitz WFJ, et al. Delayed intrauterine repair of an experimental spina bifida with a collagen biomatrix. Pediatr Neurosurg. 2007;44:29-35

21. Callens N, De Cuypere G, De Sutter P, et al. An update on surgical and non-surgical treatments for vaginal hypoplasia. Human Reproduction Update. 2014;20:75-801

22. Zhang Y, Chen Y, Hua K. Outcomes in patients undergoing robotic reconstructive uterovaginal anastomosis of congenital cervical and vaginal atresia. Int J Med Robot Comput Assist Surg. 2017;13(3):e1821

23. Shen F, Zhang XY, Yin CY, Ding JX, Hua KQ. Comparison of small intestinal submucosa graft with split-thickness skin graft for cervicovaginal reconstruction of congenital vaginal and cervical aplasia. Hum Reprod. 2016; 31:2499-2505

24. Ding JX, Chen LM, Zhang XY, Zhang Y, Hua KQ. Sexual and functional outcomes of vaginoplasty using acellular porcine small intestinal submucosa graft or laparoscopic peritoneal vaginoplasty: A comparative study. Hum Reprod. 2015; 30:581-589 
25. Huleihel L, Dziki JL, Bartolacci JG, et al. Macrophage phenotype in response to ECM bioscaffolds. Seminars in Immunology. 2017. 29:2-13

26. Zhang JK, Du RX, Zhang L, et al. A new material for tissue engineered vagina reconstruction: Acellular porcine vagina matrix. J Biomed Mater Res - Part A. 2017; 105:1949-1959

27. Zhang N, Qin X, Zhang J, et al. Bone marrow mesenchymal stem cells accelerate the morphological and functional recovery of neovaginas. Artif Organs. 2018;42(12):1206-1215

28. Lesage F, Pranpanus S, Bosisio FM, et al. Minimal modulation of the host immune response to SIS matrix implants by mesenchymal stem cells from the amniotic fluid. Hernia. 2017; 21:973-982
29. Eichler C, Schulz C, Vogt N, Warm M. The use of acellular dermal matrices (ADM) in breast reconstruction: a review. Surgical Technology International. 2017;31:53-60

30. Knabben L, Kanagalingam G, Imboden S, Günthert AR. Acellular dermal matrix (Permacol®) for heterologous immediate breast reconstruction after skin-sparing mastectomy in patients with breast cancer: A single-institution experience and a review of the literature. Front Med. 2016; 3:72

31. Ekblad Å, Fossum M, Götherström C. Soft tissue repair with easy-accessible autologous newborn placenta or umbilical cord blood in severe malformations: A primary evaluation. Stem Cells Int. 2017; 2017: 1626741

32. LoPresti ST, Brown BN. Effect of source animal age upon macrophage response to extracellular matrix biomaterials. J Immunol Regen Med. 2018; 1:57-66 